\title{
Nível de Serviços Logísticos no E-Commerce: a Percepção do Cliente para a Satisfação
}

\section{Level of Logistic Services in E-Commerce: Customer Perception for Satisfaction}

Hélio Camargos Neto*a; Lucas Beraldo Soares ${ }^{a}$; Camila Ingredy da Silva Souza ${ }^{\mathrm{a}}$; Charlles Rander e Silva ${ }^{\mathrm{a}}$; Cléber

Tiago de Souza ${ }^{a}$; Deivison Wesley Dias Silvaa; Emilly Fiama Pimentel Ferreira ${ }^{a}$; Gabriel Marques Pinheiroa; Gislaine Aparecida de Resende Pereira ${ }^{\text {a }}$ Leonardo Márcio Guedes ${ }^{\mathrm{a}}$

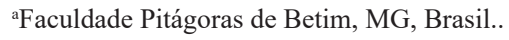

*E-mail: helio.camargos@kroton.com.br

\section{Resumo}

Com o passar do tempo, a concorrência do mercado aumenta constantemente e os clientes se tornam cada vez mais exigentes. Se as empresas não acompanharem as mudanças do mercado, poderão se tornam obsoletas, a fim de perder participação no mercado e junto uma boa parcela de sua receita. O comércio eletrônico está crescendo com o desenvolvimento dos novos modelos de negócios e será continuamente impulsionado nas próximas décadas. Com grande número de empresas atuantes no e-commerce, a logística tem sido amplamente influenciada a desempenhar um papel importante na satisfação do cliente e percepção positiva dos serviços prestados. O objetivo deste artigo é entender as percepções dos clientes em relação aos fatores de desempenho dos serviços logísticos no e-commerce. Foi relatado neste artigo um estudo exploratório através de um survey eletrônico feito pela plataforma Google Formulários tendo a participação de 303 respondentes em uma amostra não probabilística. Os resultados destacam o fato de que a dimensão relacional da logística sobre a qualidade de serviço é bem representativo sobre o nível de satisfação e taxa de retenção dos clientes. Desta forma os gestores serão capazes de correlacionar de maneira ótima as estratégias orientadas para aquisição de novos clientes e retenção de clientes existentes com a percepção de valor da qualidade do serviço logístico.

Palavras-chave: E-Commerce. Serviço Logístico. Satisfação do Cliente.

\begin{abstract}
Over time, market competition is constantly increasing and customers become increasingly demanding. If companies fail to keep up with market changes, they may become obsolete in order to lose market share and together a good share of their revenue. E-commerce is growing with the development of new business models and will be continuously boosted in the coming years. decades. With a large number of companies active in e-commerce, logistics has been largely influenced to play an important role in customer satisfaction and positive perception of the services provided. The purpose of this article is to understand the perceptions of customers regarding the factors of performance of logistics services in e-commerce. This article reports an exploratory study through an electronic survey conducted by the Google Forms platform, with the participation of 303 respondents in a non-probabilistic sample. The results highlight the fact that the relational dimension of the logistics on the quality of service is very representative about the level of satisfaction and retention rate of the customers. In this way the managers will be able to correlate in a optimal way the strategies oriented towards the acquisition of new clients and retention of existing clients with the perception of value of the quality of the logistics service.
\end{abstract}

Keywords: E-Commerce. Logistics Service; Customer Satisfaction.

\section{Introdução}

O comércio eletrônico é um comércio tradicional que acontece num ambiente eletrônico, repleto de tecnologia de comunicação e informação, buscando atender aos objetivos de negócios, sendo considerado de fácil acesso e baixo custo. Trata-se de uma realidade em diversos setores da economia, estando em um estágio de evolução intermediário. O cenário, mesmo que não uniforme para todas as empresas em um índice ainda não muito elevado, permite avaliar tal evolução e identificar as tendências do mercado eletrônico (ALBERTIN, 2010).

Em razão da globalização, qualquer empresa que decidir utilizar a internet para comercialização de seus produtos deve estar ciente de que enfrentará 24 horas por dia a necessidade de disponibilidade de sua proposta para com seu público-alvo, em que todos os setores da empresa devem estar preparados para o pronto atendimento (UEMA; LAZZARI, 2008). Para Souza, Lemos e Zorgo (2014) o comercio eletrônico passou a representar para a logística, dimensões mais amplas, pois, cada acesso ao sistema pode gerar um novo pedido, criando necessidades de transporte e distribuição que se diferenciam do comercio tradicional dada sua abrangência geográfica.

De acordo com Ballou (2001) a missão da logística é dispor a mercadoria/serviço certo, no lugar e tempo certos e nas condições desejadas, ao mesmo tempo em que fornece maior contribuição à empresa. No e-commerce a logística busca se preocupar com a agilidade de entrega, cumprindo prazos, e se possível, efetuando a entrega com o tempo menor do que o prometido.

O serviço ao cliente é o componente que diferencia a logística moderna da abordagem tradicional, atualmente a importância não reside somente na busca pela eficiência 
operacional, mas também pelo atendimento das necessidades dos clientes (SILVA; LOOS, 2017). Se houver falha no serviço de entrega, cabe ao funcionário da empresa responder às reclamações, sendo o conteúdo e a forma de resposta que determinante para a percepção de satisfação ou não dos clientes (ARAÚJO et al. 2012).

O construto lealdade deve ser encarado como opção para o desenvolvimento e estabelecimento de vantagem competitiva, independente do segmento no qual esteja inserido a organização. Nesta perspectiva a satisfação e lealdade devem ser priorizadas visando alcance e maximização dos resultados. Cabe ainda ressaltar, que o papel da Logística é buscar, através de um nível de serviço, a satisfação e lealdade dos clientes.

Desta forma, o objetivo deste artigo é apresentar a importância do nível de serviços logísticos para o e-commerce demonstrando sua relevância para satisfação e tendo como consequente, a lealdade.

\section{Material e Métodos}

Para coleta dos dados optou-se, por um questionário estruturado, em escala tipo likert contendo 23 perguntas, enviados por e-mail e whatsapp, visando obter a percepção do cliente quanto a confiabilidade do prazo e agilidade na entrega, rastreabilidade do produto, comunicação entre empresa e consumidor, melhor preços e condições, qualidade do produto entregue.

Os dados foram coletados por meio desse questionário estruturado em escala de cinco pontos, este método consiste em "um conjunto de itens apresentados como afirmações ou opiniões, para os quais se pede a reação dos participantes" em relação ao objeto investigado (SAMPIERI; COLLADO; LUCIO, 2013, p. 261). Para relacionar o grau de concordância entre os entrevistados foi utilizada a escala entre: 1) discordo totalmente, 2) discordo, 3) indiferente (ou neutro), 4) concordo e 5) concordo totalmente compreendendo os assuntos relacionados à percepção do cliente quanto ao uso do E-commerce.

O referido estudo trata-se um estudo de caso, cujo propósito é reunir informações detalhadas e sistemáticas sobre um fenômeno (PATTON, 2002). O estudo de caso possui características próprias e pode ser conceituado com base nas posições de dois dos mais reconhecidos especialistas neste método (YIN, 2002; STAKE 1995).

\section{Resultados e Discussão-}

\subsection{E-Commerce}

Para Albertin (2000) o comércio eletrônico é a realização de toda a cadeia de valor dos processos de negócio, num ambiente eletrônico, pela aplicação das tecnologias de comunicação e informação, atendendo aos objetivos do negócio. Além de Comércio Eletrônico esse tipo de transação comercial feita exclusivamente por meio de um equipamento eletrônico tem outros nomes, como: E-commerce e comércio virtual (ALMEIDA; BRENDLE; SPINDOLA, 2014). O e-commerce teve início em 1995, nos Estados Unidos, com o surgimento da Amazon.com e outras empresas. E, só após cinco anos, esse setor começou a se desenvolver no Brasil. Desde então, as vendas pela internet não param de crescer (TOREZANI, 2008). Albertin (2000) já afirmava que o comércio eletrônico no mercado brasileiro estava tendo claros sinais de evolução.

O Comércio eletrônico ou e-commerce tem se tornado uma ferramenta tecnológica essencial para as empresas na atualidade por se tornar um diferencial no mercado e alavancar a competitividade da empresa (PRADO; PEREIRA; GUELLER, 2016). Entende-se que o e-commerce possibilita que as empresas comercializem seus produtos e serviços sem que seus consumidores saiam do conforto de suas casas, pois os mesmos passaram a ser mais exigentes e buscam atingir suas expectativas realizando suas compras de forma online de onde quer que estejam.

A internet é uma ferramenta que permite fácil busca de produtos, o que facilita a comparação de preços, sendo este o critério mais impactante de decisão de compra. A segurança, porém, vem se tornando tema importante nas compras online.

Peixoto (2001) ressaltou que as empresas que praticam comércio eletrônico devem estar preparadas para o atendimento legal das normas de proteção ao consumidor, investindo em mecanismos tecnológicos aptos a promover a efetiva defesa dos direitos dos usuários no ambiente eletrônico.

\subsection{Logística}

A logística surgiu nos tempos da Segunda Guerra Mundial, onde os militares necessitavam de estratégias para combater seus inimigos, desta forma os generais definiam equipes que controlavam os transportes de materiais como munição, medicamentos, equipamentos que utilizavam. Assim o uso dessas estratégias era imprescindível naquela época (NOVAES, 2007). Segundo Cavanha Filho (2001), a Logística pode ser definida como a parte do processo da cadeia de suprimento que planeja, implementa e controla o eficiente e efetivo fluxo e estocagem de bens, serviços e informações relacionadas, do ponto de origem ao ponto de consumo, visando atender aos requisitos dos consumidores.

Nos primeiros estudos, o uso desse termo buscou enfatizar a redução dos estoques dentro e por meio das empresas, sendo tal perspectiva ampliada posteriormente, abordando a gestão da cadeia de suprimentos como uma logística por meio das fronteiras interorganizacionais (COOPER; LAMBERT; PAGH, 1997; MACBETH; FERGUSON, 1993). Christopher (2009) complementa que a logística é responsável por gerenciar estrategicamente a aquisição, o transporte e a armazenagem de matérias-primas, componentes e produtos acabados, além dos fluxos de informação relacionados. 


\subsection{Logística no e-commerce}

O comercio eletrônico passou a representar para a logísticas dimensões mais amplas, pois cada acesso ao sistema pode gerar mais um novo pedido, criando necessidades de transporte e distribuição que se diferenciam do comercio tradicional dada sua abrangência geográfica (SOUZA; LEMOS; ZORGO, 2014).

Na visão de Uehara (2001), o processo de atendimento do pedido e a distribuição são os grandes entraves no comércio eletrônico. Para Lacerda (2000) as compras são feitas com maior frequência, em pequenas quantidades, gerando uma entrega fracionada e aumentando a importância de disponibilizar produtos em tempo.

Fleury e Monteiro (2000) apresentam um quadro com as principais diferenças entre a logística tradicional e a logística utilizada em rede no Comércio Eletrônico:

Quadro 1 - Diferenças entre logística tradicional e logística do E-commerce

\begin{tabular}{|c|c|c|}
\hline & $\begin{array}{c}\text { Logística } \\
\text { Tradicional }\end{array}$ & $\begin{array}{c}\text { Logística do } \\
\text { Comércio } \\
\text { Eletrônico }\end{array}$ \\
\hline $\begin{array}{c}\text { Tipo de } \\
\text { carregamento }\end{array}$ & Paletizado & Pequenos Pacotes \\
\hline Clientes & Conhecidos & Desconhecidos \\
\hline $\begin{array}{c}\text { Tamanho médio do } \\
\text { pedido }\end{array}$ & Mais de US\$ 1.000 & Mais de US\$100 \\
\hline $\begin{array}{c}\text { Destino dos } \\
\text { pedidos }\end{array}$ & Concentrados & $\begin{array}{c}\text { Altamente } \\
\text { Dispersos }\end{array}$ \\
\hline $\begin{array}{c}\text { Responsabilidade } \\
\text { Um Único Elo }\end{array}$ & $\begin{array}{c}\text { Toda Cadeia de } \\
\text { Suprimento }\end{array}$ \\
\hline Demanda & $\begin{array}{c}\text { Estável e } \\
\text { Consistente }\end{array}$ & $\begin{array}{c}\text { Incerta e } \\
\text { Fragmentada }\end{array}$ \\
\hline
\end{tabular}

Fonte: Fleury e Monteiro (2000).

Segundo Novaes (2004), muitas empresas se surpreendem pelo número de pedidos, após terem utilizado o comércio eletrônico para comercializar seus produtos, e muitas vezes um número acima de sua capacidade comercial, sendo assim todos os setores de sua empresa deverão estar preparados para um pronto atendimento (UEMA; LAZZARI, 2008).

Conforme Kotler e Keller (2012) as empresas dependem dos clientes, sendo necessário investir em qualidade, uma vez que, com clientes exigentes o preço não é mais o único fator de compra, a qualidade em serviços é um componente central das percepções dos clientes, fazendo que os mesmos se fidelizem a empresa voltando a realizar novas compras.

\subsection{Logística: satisfação do cliente}

No caso e-commerce, essencialmente, o cliente potencial é impulsionado a comprar através da Internet por diversos motivos, dando grande importância aos fatores logísticos, fazendo com que a empresa dê atenção especial à infraestrutura logística, associando estoques, distribuição, tratamento da informação, recursos humanos etc. (NOVAES, 2007). A partir do instante em que a produção é finalizada até o momento no qual o consumidor tem acesso ao produto final, as mercadorias tornam-se responsabilidade da logística, que deve-se preocupar e garantir que os produtos desejados pelos clientes estejam disponíveis à medida que são solicitados, considerando a ideia do custo razoável (BALLOU, 2010). Para Kotler e Keller (2012) a satisfação é a comparação feita por uma pessoa sobre o desempenho percebido de um produto em relação as suas expectativas, quando o desempenho não atinge as expectativas, o cliente fica decepcionado, mas se o desempenho alcançar as expectativas, o cliente fica satisfeito. Já quando o desempenho supera as expectativas, o cliente fica encantado.

O serviço ao cliente é o componente que diferencia a logística moderna da abordagem tradicional, já que atualmente a importância não reside somente na busca pela eficiência operacional, mas também pelo atendimento das necessidades dos clientes (FLEURY; WANKE; FIGUEIREDO, 2000). A função logística busca oferecer um serviço ao cliente de qualidade superior e de baixo custo, gerando valor para os clientes.

A satisfação do consumidor está ligada exclusivamente aos serviços logísticos, o objetivo é fornecer utilidade de tempo e lugar na transferência de produtos e serviços entre comprador e vendedor. Mais especificamente, são sete os objetivos do serviço logístico, denominado de sete R's (sete condições corretas para o serviço logístico): entregar o produto certo, na quantidade certa, dentro do tempo de entrega requisitado, na condição certa (sem avarias), para o cliente certo, no local especificado e a um custo aceitável (CHRISTOPHER, 1997; FLEURY, WANKE; FIGUEIREDO, 2000; BALLOU, 2001; COLLINS; HENCHION; O'REILLY, 2001; STANK et al., 2003; RAFELE, 2004). Na comercialização eletrônica, a forma e o tempo de entrega dos produtos para o consumidor final são fatores determinantes para sua conquista e fidelização, fazendo com que a logística seja uma área de grande valor e de importância imediata para aqueles que fazem parte do e commerce.

Conforme Anderson et al. (1994) a satisfação do consumidor pode ser conceituada a partir de duas diferentes perspectivas: [i] transação-específica, em que a satisfação é vista como um julgamento avaliativo de uma específica ocasião de compra e cumulativa, onde a satisfação é definida como uma avaliação geral baseada em sucessivas compras. Para Mentzer, Flint e Hult (2001), entregar ao cliente um produto livre de defeitos, de forma mais rápida e mais segura que a concorrência, não é mais visto como uma vantagem competitiva, mas simplesmente uma exigência para estar no mercado.

Existindo uma falha no serviço de entrega, cabe ao funcionário da empresa responder às reclamações, e é justamente o conteúdo e a forma de resposta que determinam a percepção de satisfação ou não dos clientes (BITNER; BOOMER; TETREALUT, 1990).

A resolução de discrepâncias no pedido se refere a quão 
bem o fornecedor lida com as diferenças em pedidos quando estas forem realizadas. $\mathrm{O}$ cliente espera que, se algo venha errado com seu pedido, isso seja corrigido rapidamente e de forma adequada. Isto afeta sua percepção de qualidade quanto ao fornecedor. (MENTZER; FLINT; HUNT, 2001).

A relação entre qualidade e satisfação procede da teoria do paradigma da desconformidade, sugerindo a percepção da qualidade como uma das variáveis formadoras da satisfação (OLIVER, 1999). A qualidade é um determinante da satisfação e que possui consequências potenciais para influenciar a lealdade (DICK; BASU, 1994, ZEITHAML; BERRY; PARASURAMAN, 1996).

\subsection{Lealdade}

Em uma busca na literatura sobre lealdade, é possível encontrar mais de 20 definições para este construto (DAVIS; MENTZER, 2008). A grande dificuldade para o consenso está nas diferentes visões do que compõe a lealdade. Saura et al. (2008) argumentam que existem duas visões para lealdade, a primeira focando apenas na retenção do cliente e sua compra contínua na empresa, enquanto a segunda inclui uma dimensão afetiva, sendo a lealdade o resultado deste elo emocional entre o cliente e a marca.

A lealdade é uma atitude positiva, que envolve vínculos emocionais e de continuidade de relacionamento (MOWEN; MINOR, 2003, SIRDESHMUKH; SINGH; SABOL, 2002; BLOEMER; RUYTER; WETZELS, 1999). O comportamento definido como lealdade representa não apenas o significado Comportamental, caracterizado pela repetição da compra, mas também significado Psicológico (OLIVER, 1999). De acordo com essa visão, não é correto fazer qualquer inferência sobre lealdade apenas com base em Padrões de compra repetida, uma vez que a lealdade verdadeira inclui, além do Comportamento, uma resposta atitudinal, constituída por componentes cognitivos se afetivos. Esses componentes apresentam dimensões como acessibilidade, Confiabilidade, emoções e sentimentos, caracterizando a lealdade como construto Multidimensional (DICK; BASU, 1994).

Para Dick e Basu (1994) a lealdade representa mportante base para desenvolver vantagem competitiva sustentável, porém, devido à acirrada concorrência e volatilidade do mercado, sua gestão tornou-se um desafio para as empresas. Para que o consumidor se torne (ou mantenha-se) leal à marca deve haver a crença de que o produto e/ou serviço da empresa é (ou continua a ser) a melhor alternativa do mercado (OLIVER, 1999).

\subsection{Discussão}

Após a aplicação de um questionário estruturado na escala "Likert", voltada a satisfação do cliente no e-commerce, dos quais houveram 303 respondentes. Chegou-se aos seguintes dados e conclusões: $85,1 \%$ já realizaram compras pela internet, $14,2 \%$ nunca realizaram, sendo constatado que a maioria dos compradores com a faixa etária entre 25 a 34 anos corresponde há $38,1 \%, 18$ a 24 anos a $25,4 \%, 35$ a 44 a anos $24,6 \%$ e de 45 a 54 anos a 8,8\%. Dos participantes $61,2 \%$ são do gênero masculino e $38,1 \%$ são do gênero feminino.

O nível de escolaridade dos entrevistados graduados é de $48,8 \%$, ensino médio $27,7 \%$, pós-graduados $16,5 \%$ e $11 \%$ possuem ensino fundamental. Após a análise dos dados constatou-se que a maior parte das compras realizadas no e-commerce é direcionada ao setor de eletrônicos.

Entretanto, este novo consumidor ainda configura um novo desafio para os varejistas. As crenças, os hábitos e as práticas mudaram. Agora os clientes controlam o processo de compra. Várias compras virtuais são realizadas antes dos clientes sequer visitar as lojas físicas.

De acordo com análise realizada na pesquisa conclui-se que a entrega com rapidez e no prazo estipulado, sem divergências ou defeitos, tanto no produto como nas embalagens é de suma importância para gerar mais confiabilidade para futuras compras, uma vez que, o recebimento do produto com embalagem danificada no processo de transporte gera má impressão. Neste caso a organização poderá incorrer em propagandas boca a boca desfavoráveis e obter avaliações negativas devido a insatisfação do cliente, que poderão ainda, depor contra a sua imagem.

Segundo os respondentes, o preço do produto na loja virtual incluindo o valor do frete é melhor que as compras feitas em lojas físicas. O poder do e-commerce mostra-se pujante e robusto, uma vez que os clientes já percebem as vantagens de efetuar as compras no conforto de casa, pelo de seu smartphone, onde é permitido pelo e-commerce, fazer comparações em relação aos produtos a serem adquiridos.

No entanto, cabe ressaltar que, caso o produto não seja entregue dentro do prazo determinado e na qualidade esperada gera grande insatisfação e má credibilidade com o site por parte do cliente. Dessa forma, é muito importante para os consumidores que após realizada a compra exista contato por parte da empresa com intuito de esclarecer as dúvidas, reclamações para que o cliente se sinta bem atendido (pósvenda estruturado), pois o mesmo realiza pesquisa sobre confiabilidade e credibilidade das empresas antes de efetuarem as compras online.

Ressalta-se que existe preocupação por boa parte dos consumidores com o método de transporte da mercadoria, pelo fato de terem a percepção que os mesmos influenciam diretamente no prazo de entrega. Segundo a pesquisa, o consumidor tem se sentido satisfeito quanto aos prazos de entrega dos produtos, assim como julgam importante a possibilidade de rastreamento do produto, vez que possibilita saber em que parte do processo logístico ele se encontra, trazendo assim mais tranquilidade e menos ansiedade acerca da data de sua entrega. Novamente podemos observar quanto o cliente assume um poder mandatário, ou seja, possui a incumbência de ter uma posição ativa e determinante no processo decisorial de compra via e-commerce. 


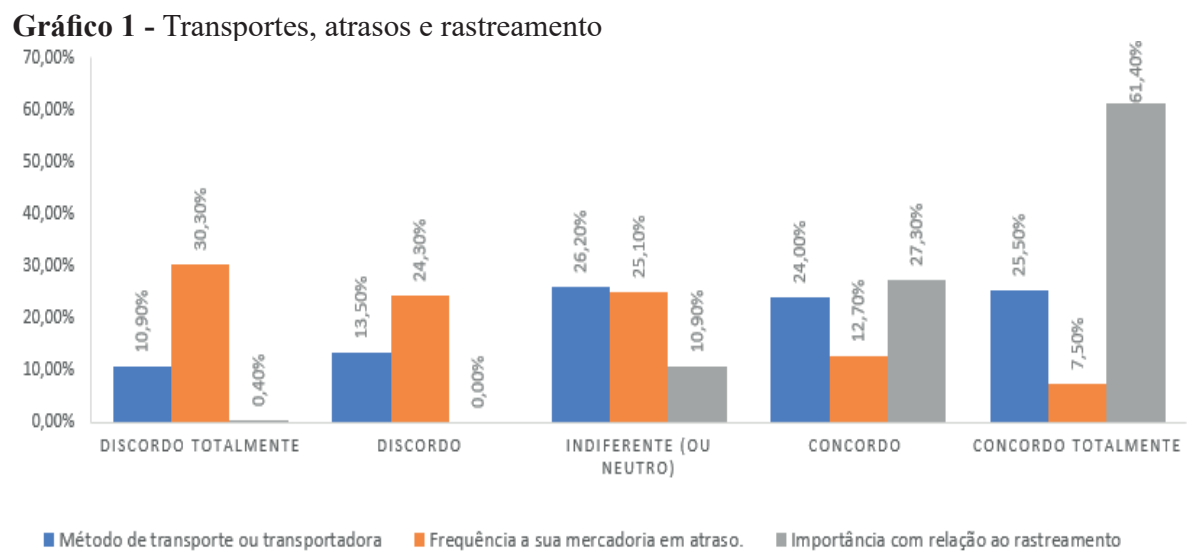

Fonte: Dados da pesquisa.

A pesquisa realizada também revelou que a predileção dos consumidores pela venda via e-commerce decorre da maior variedade de produtos ofertada em relação ao comércio tradicional (lojas físicas). Além da variedade e da comodidade de realizar a compra em qualquer lugar e horário, incluindo no conforto de seus lares, o preço do produto ainda é o fator determinante para a realização das compras via e-commerce, mas alguns clientes estariam dispostos a pagar um valor de frete a mais para que o produto chegasse mais rápido, devido à urgência em poder usufruir do produto adquirido. Conforme pode ser observado, o cliente torna-se cada vez mais influenciador. Expressa, amplia e apregoa rapidamente via networking suas experiências, críticas e, desta forma, um atendimento superior traz inúmeras vantagens à organização.

Gráfico 2 - Variedades, baixo custo

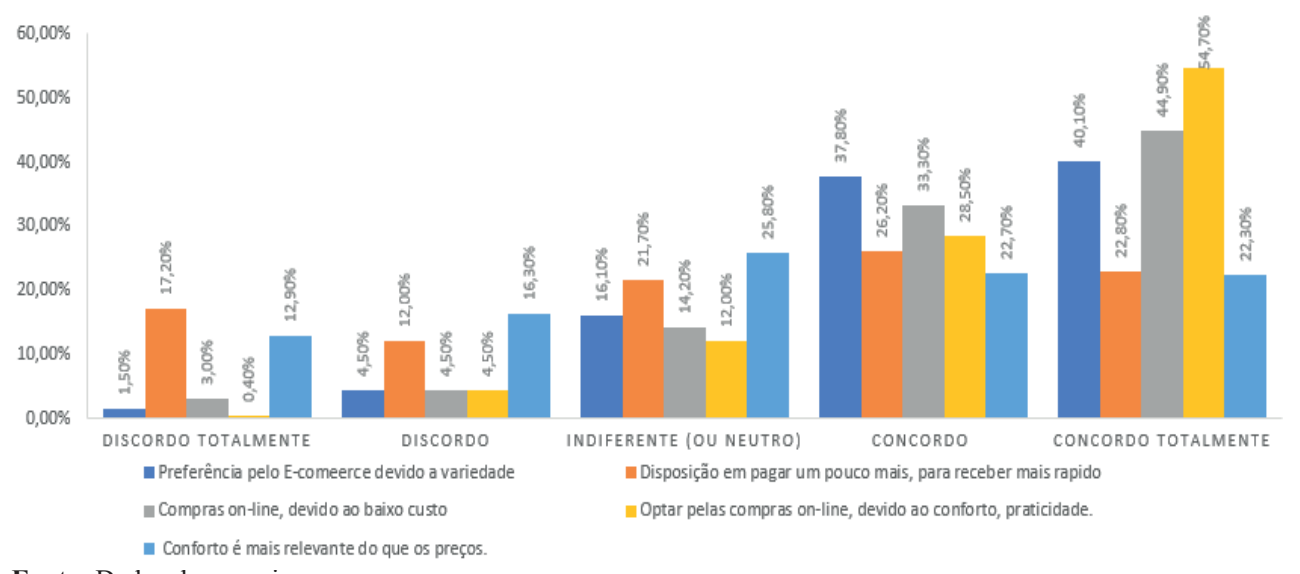

Fonte: Dados da pesquisa.

A maioria dos consumidores realiza suas compras em smartphones através de aplicativos de lojas ao invés de realizá-las em computadores, o que confirma a praticidade e o crescimento deste tipo de comércio, que atrelado a um bom atendimento e um bom processo logístico vem conquistando a preferência dos consumidores para compras on-line.

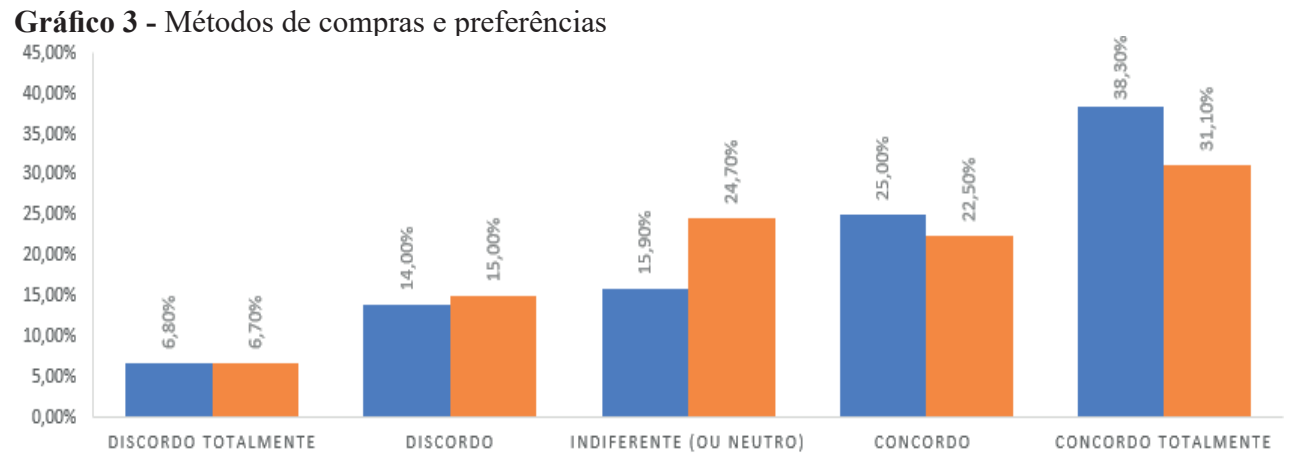

a Compras pelo aplicativo ou sites a Preferencia em compras on-line, superior a compras tradicionais

Fonte: Dados da pesquisa. 
A variedade de produtos é outro atrativo que o e-commerce proporciona, ou seja, um site com boa variedade de produtos cria maior possibilidade de aumento no ticket médio de compra e pode evitar o abandono de carrinhos vazios.

\section{Conclusão-}

Com o surgimento da internet a alguns anos atrás, vieram juntamente vários avanços tecnológicos que proporcionaram facilidade e comodidade para seus usuários. Uma dessas inovações foi o surgimento do e-commerce (comercio eletrônico), que se tornou com o tempo, fundamental para as empresas, por se tornar uma ferramenta tecnológica essencial para aumentar a sua visibilidade e competitividade no mercado.

Por sua vez, o consumidor sempre ligado as evoluções e inovações tecnológicas encontraram no e-commerce uma ferramenta moderna e prática para conhecer produtos variados, pesquisar sobre as empresas que os oferecem, comparar preços e custo benefício com comodidade, de forma rápida e pratica através de computadores, smartphones a qualquer hora e em qualquer lugar no mundo, devido a escassez de tempo para se deslocarem até uma loja física.

As empresas perceberam que não adianta oferecer apenas um bom preço aos seus clientes, pois os mesmos ao realizarem uma compra, criam expectativas de receber um bom produto e passar por uma boa experiência, que vai desde o primeiro contato com site até o recebimento da mercadoria em sua casa com qualidade e integridade do produto.

Tendo em vista as novas demandas e exigências dos consumidores, as empresas veem adotando várias medidas para atender a essas expectativas. Dentro deste contexto a logística tem se mostrado de suma importância para solucionar todos os desafios relacionados a esse processo, pois ela é o elo que liga toda a cadeia de suprimentos que vem da percepção do consumidor final do qual se extrai a previsão de demanda. A logística trata da vertente que irá agregar valor de forma positiva, será capaz de criar e manter vantagem competitiva sustentável para as organizações a que conduzirem de forma estratégica e responsiva.

No mercado competitivo atual, a empresa que busca modernizar e aprimorar o processo logístico tem um grande diferencial no mercado, pois é ele que torna possível todo o processo de compra, transporte e entrega do produto, uma vez que, sem a logística nada disso seria possível.

Considerando-se a pesquisa realizada e os fatores citados acima, nota-se que a grande maioria das pessoas tem preferência em realizar suas compras pelo comercio eletrônico, pois os clientes tem se sentido bastante satisfeito com o retorno dos serviços prestados pelas empresas de e-commerce, pelo fato de estarem investido constantemente na evolução do processo logístico em geral, a compra on-line adquiriu um nível bastante elevado em relação à satisfação dos clientes, devido à grande concorrência no mercado a empresa que oferecer o melhor serviço terá um grande diferencial, pelo fato do consumidor realizar uma pesquisa previa antes de efetivar suas compras, ele leva em consideração a boa relação da empresa com o cliente, sua classificação e sua boa índole no mercado.

\section{Referências}

ALBERTIN, A.L. O comércio eletrônico evolui e consolida-se no mercado brasileiro. RAE, v.40, n.4, p.94-102, 2000.

ALBERTIN, A.L. Comércio eletrônico: modelo, aspectos e contribuições de sua aplicação. São Paulo: Atlas, 2010.

ALMEIDA, R.E.S.; BRENDLE, V.; SPINOLA, N.D. Ecommerce: evolução, processo de compra, e o desafio da entrega. $R D E$, v.16, n.29, p.138-149, 2014.

ANDERSON, E. et al. Customer satisfaction, market share and profitability: findings from sweden. J. Marketing, n.58, p.53-66, 1994.

ARAÚJO, F.F. et al. A qualidade do serviço de logística como vantagem competitiva: uma pesquisa no mercado de iogurtes de Belém. In: ENCONTRO DA ANPAD, 36. Rio de Janeiro, 2012. Disponível em: http://www.anpad.org.br/diversos/down zips/63/2012_GOL1859.pdf Acesso em: 11 mar. 2019.

BALLOU, R.H. Gerenciamento da cadeia de suprimentos: planejamento, organização e logística empresarial. Porto Alegre: Bookman, 2001.

BALLOU, R.H. Logística empresarial: transportes, administração de materiais e distribuição física. São Paulo: Atlas, 2010.

BITNER, M.J; BOOMS, B.H.; TETREAULT, M.S. The Service Encounter: Diagnosing Favorable and Unfavorable Incidents. J. Marketing. v.54, p.71-84, 1990.

BLOEMER, J.; RUYTER, K.; WETZELS, M. Linking perceived service quality and service loyalty: a multi-dimensional perspective. Euro. J. Marketing, v.33, n.11/12, p.1082-1106, 1999.

CAVANHA FILHO, A. Logística: novos modelos. Rio de Janeiro: Qualitymark, 2001.

CHRISTOPHER, M. Logística e gerenciamento da cadeia de suprimentos: criando redes que agregam valor. São Paulo: Cengage Learning, 2009

CHRISTOPHER, M. Logística e gerenciamento da cadeia de suprimentos: estratégias para a redução de custos e melhoria dos serviços. São Paulo: Pioneira, 1997.

COLLINS, A.; HENCHION, M.; O'REILLY, P. Logistics customer service: performance of Irish food exporters. Int. J. Retail \& Distribution Management, v.29, n.1, p.6-15, 2001.

COOPER, M.C.; LAMBERT, D.M.; PAGH, J.N. Supply chain management: more than a new name for logistics. Int. J. Logistics Management, v.8, n.1, 1997.

COSTA, C.U.; NAKATA, Y.U.; CASLSANI, J.R,S. Qualidade no Atendimento: A influência do Bom Atendimento para Conquistar os Clientes. Rev. Cient. Eletr. UNISEB, v.1, n.1, p.54-65, 2013.

DAVIS, D., MENTZER, J. Relational resources in interorganizational exchange: the effects of trade equity and brand equity. J. Retailing, v.84, n.4, p.435-448, 2008.

DICK, A.S.; BASU, K. Customer loyalty: toward an integrated conceptual framework. J. Acad. Marketing Scie., v.22, n.2, p.99113, 1994.

FLEURY, P.F.; MONTEIRO, J.R.C. O desafio logístico do e-commerce. Rio e Janeiro: Centro COPPEAD. UFRJ, 2000. 
FLEURY, P. F.; WANKE, P.; FIGUEIREDO, K.F. Logística empresarial: a perspectiva brasileira. São Paulo: Atlas, 2000.

KOTLER, P.; KELLERM K.L. Administração de Marketing. São Paulo: Pearson Education do Brasil, 2012.

LACERDA, L. Logística empresarial: a perspectiva brasileira. São Paulo: Atlas, 2000.

MACBETH, D.K.; FERGUSON, N. Partnership sourcing: an integrated supply chain management approach. London: Pitman Publishing, 1993

MOWEN, J.C.; MINOR, M.S. O comportamento do consumidor. São Paulo: Pearson Prentice Hall, 2003.

NOVAES, A.G. Logística e gerenciamento da cadeia de distribuição: estratégia, operação e avaliação. Rio de Janeiro, Elsevier, 2004.

NOVAES, A.G. Logística e gerenciamento da cadeia de distribuição: estratégia, operação e avaliação. Rio de Janeiro: Elsevier, 2007.

OLIVER, R.L. Whence consumer loyalty? J. Marketing, v.63, p.33-44, 1999.

PATTON, M.G. Qualitative research and evaluation methods. Thousand Oaks: Sage, 2002.

PRADO, A.R.M.; PEREIRA, G.L.; GUELLER, J.R.L. E-commerce B2B como estratégia de internacionalização: estudo de caso de uma empresa Taiwanesa. Observatorio de la Economía Latinoamericana, 2016. Disponível em: http://www.eumed.net/ cursecon/ecolat/br/16/b2b.html Acesso em: 11 ago. 2019.

PEIXOTO, R.C. O comércio eletrônico e os contratos. Rio de Janeiro: Forense, 2001.

RAFELE, C. Logistic service measurement: a reference framework. J. Manufacturing Technol. Management, v. 24, n.3, p.280-290, 2004.
SAMPIERI, R.H.; COLLADO, C.F.; LUCIO, M.P.B. Metodologia de pesquisa. Porto Alegre: Penso, 2013.

SAURA, I.G. et al. Logistics service quality: a new way to loyalty. Ind. Manag. Data Systems, v.108, n.5, p.650-668, 2008

SILVA, J.A.S.; LOOS, M.J. As melhorias no processo de distribuição logística por meio do planejamento de aderência de tempo. Rev. Espacios, v.38, n.19, p.34-45, 2017.

SIRDESHMUKH, D.; SINGH, J.; SABOL, B. Consumer trust, value, and loyalty in relational exchanges. J. Marketing, v.66, p.15-37, 2002

STAKE, R.E. The art of case study research. Thousand Oaks: SAGE, 1995.

STANK, T. et al. Logistics service performance: estimating its influence on market share. J. Bus. Logistics, v.24, n.1, p.27-55, 2003.

TOREZANI, N. O crescimento do e-commerce no Brasil. Rev. iMasters, 2008. Disponível em: http://imasters.com.br/ $\operatorname{artigo/9649/e-commerce/o-crescimento-do-e-commerce-no-~}$ brasil/.

Acesso em: 15 ago. 2019.

UEHARA, L. Evolução do desempenho logístico no varejo virtual do Brasil. Rio de Janerio: COPPEAD, 2001.

UEMA, E.; LAZZARI, C.P. O crescimento do e-commerce no Brasil: estudo de caso do "Submarino". Rev. Cient. Fac. Américas, n.2, 2008.

YIN, R.K. Case study research: design and methods. Thousand Oaks: SAGE, 2002.

ZEITHAML, V.A; BERRY, L.L.; PARASURAMAN, A. The behavioral consequences of services quality. J. Marketing. v.60, p.31-46, 1996. 